\title{
MULTIPOLE MOMENTS OF STELLAR OSCILLATION MODES
}

\author{
ANDREAS REISENEGGER ${ }^{1}$ \\ Institute for Advanced Study, Princeton, NJ 08540² \\ and \\ California Institute of Technology, Pasadena, CA 91125
}

\begin{abstract}
$A B S T R A C T$
The oscillating mass $2^{l}$-pole moment, $M_{n l}$, of a star in a given (normalized) oscillation mode determines the energy that can be absorbed by the mode in a tidal interaction and the power radiated by the mode in gravitational waves, both of which are proportional to $\left|M_{n l}\right|^{2}$. The coefficients in the expansion of the vector fields $\nabla\left[r^{l} Y_{l m}(\theta, \phi)\right]$ in terms of the displacement fields of modes of given $l$ and $m$ are (proportional to) these multipole moments. This expansion leads to a sum rule $\sum_{n}\left|M_{n l}\right|^{2}=$ constant. For stars of weak to moderate central condensation (such as neutron stars), the f-mode is well approximated by the vector field being expanded, and therefore it takes the lion's share of the sum. Thus, the multipole moments of all other modes must be small. In their numerical evaluation, it is necessary to know the shape of the eigenfunctions quite precisely, since a small $f$-mode contamination can significantly increase the obtained values. The implications of these results for computations of oscillation modes from Newtonian equations in relativistic neutron star models are discussed in detail.
\end{abstract}

Subject headings: radiation mechanisms: gravitational — relativity - stars: neutron stars: oscillations

1 E-mail: andreas@guinness.ias.edu

2 Present address. 


\section{INTRODUCTION}

The study of nonradial oscillation modes in Newtonian stars has a long and rich history, going back to the pioneering work of Kelvin (Thomson 1863), Pekeris (1938), and Cowling (1941). The knowledge accumulated from this work has been summarized in the books by Cox (1980) and Unno et al. (1989).

Nonradial oscillation modes can be excited (among other possibilities) when the star suffers a tidal interaction with another object (Cowling 1941; Zahn 1970), which might be passing or captured in a binary orbit. In such an interaction, the modes absorb energy from the relative motion of the two objects, leading to tidal capture (Fabian, Pringle, \& Rees 1975; Press \& Teukolsky 1977), orbital circularization, modification of the stellar (differential) rotation (Zahn 1975, 1977; Goldreich \& Nicholson 1989), or accelerated inspiral of a close binary (Reisenegger \& Goldreich 1994). The amount of energy absorbed depends on the overlap of the tidal force field with the displacement field of the mode in question or, equivalently, on the appropriate multipole moment of the mode.

The nonradial oscillation modes of relativistic stars have also been studied in detail (Thorne \& Campolattaro 1967; Price \& Thorne 1969; Thorne 1969a, b; Campolattaro \& Thorne 1970; Ipser \& Thorne 1973; Detweiler \& Ipser 1973). The most important new effect that appears in this case is the emission of gravitational radiation by the oscillating star. This effect makes the calculations much more complicated and less transparent. Other relativistic effects are usually not overwhelmingly important in cases of astronomical interest such as neutron stars, at least not if compared to the uncertainties introduced by our lack of knowledge of the physics of matter at high densities. This has motivated many authors to compute the mode eigenfunctions and frequencies in a purely Newtonian framework, and to evaluate the damping due to the emission of gravitational waves from the so-called "quadrupole formula" and its extensions to higher multipoles (Thorne 1969b; Balbinski \& Schutz 1982). This formula gives the radiated power in terms of a multipole moment of the mode. It is correct to lowest post-Newtonian order (see Damour 1987 for a full discussion). Some authors (Balbinski \& Schutz 1982; McDermott 1985; McDermott, Van Horn, and Hansen 1988; Strohmayer 1991; Strohmayer et al. 1991) have also computed modes by applying Newtonian dynamical equations to a relativistic stellar equilibrium model. In these "hybrid" models, the "quadrupole formula" must also be used for the evaluation of the gravitational wave emissivity.

The general purpose of the present paper is to study the multipole moments of stellar oscillations, whose physical significance was outlined in the previous two paragraphs, but which appear not to have attracted considerable attention in the past. In $\S 2$, it is pointed out that the oscillation operator, whose eigenfunctions are the Newtonian modes, is Hermitian, even in the often used Cowling approximation. This implies (§3) that the modes are orthogonal, and that (probably) any "smooth" vector field can be written as a linear combination of all modal displacement fields. For each multipole order $l$, there is a simple vector field for which the expansion coefficients are the $2^{l}$-pole moments of the modes. This leads (for each $l$ ) to a sum rule for the squared amplitudes of the $2^{l}$-pole moments. For stellar models that are not very centrally condensed, most of the contribution to the sum comes from the f-mode (of the corresponding $l$ ), whose displacement field is very similar 
to the vector field being expanded $(\S 4)$. This implies that the multipole moments of all other modes must be quite small, with obvious implications for the applications discussed in the previous paragraphs. In $\S 5$, I point out that, although the general-relativistic oscillation operator is not Hermitian (its eigenvalues have imaginary parts that account for the damping due to gravitational radiation), its eigenfunctions are still orthogonal. Thus, the Hermiticity of the operator should be preserved in the hybrid models in order to give a good approximation to the correct (fully relativistic) ones. The fact that this is not the case for some of the mode computations in the literature has important consequences for the computed damping times $(\S 6)$. The main conclusions of this work are given in $\S 7$.

\section{HERMITICITY OF THE OSCILLATION OPERATOR}

The displacement fields $\boldsymbol{\xi}_{n l m}(\mathbf{x})$ associated with the oscillation modes of spherically symmetric stars in Newtonian mechanics are eigenfunctions of a linear "oscillation operator" $\mathcal{H}$ (e.g., Cox 1980; Lynden-Bell \& Ostriker 1967), with the boundary condition

$$
\left.\boldsymbol{\nabla} \cdot \boldsymbol{\xi}\right|_{r=R}=0
$$

where $R$ is the stellar radius. This condition is equivalent to demanding that the Lagrangian pressure perturbation vanish at the surface.

The oscillation modes come in two varieties, spheroidal modes of the form

$$
\boldsymbol{\xi}_{n l m}(\mathbf{x})=\xi_{n l}^{r}(r) Y_{l m}(\theta, \phi) \hat{\mathbf{r}}+\xi_{n l}^{\perp}(r) r \nabla Y_{l m}(\theta, \phi)
$$

and toroidal modes,

$$
\boldsymbol{\xi}_{n l m}(\mathbf{x})=\xi_{n l}^{t}(r) r \hat{\mathbf{r}} \times \nabla Y_{l m}(\theta, \phi)
$$

(Aizenman \& Smeyers 1977). Here, $n$ is an arbitrary label for the radial eigenfunctions, $Y_{l m}(\theta, \phi)$ are the standard spherical harmonics (e.g., Jackson 1975), and the functions of radius $\xi_{n l}^{r}(r), \xi_{n l}^{\perp}(r)$, and $\xi_{n l}^{t}(r)$ depend on the stellar model chosen. ${ }^{3}$

It is convenient to define the inner product,

$$
\langle\boldsymbol{\zeta} \mid \psi\rangle \equiv \int_{\star} \rho_{0}(r) \boldsymbol{\zeta}^{*}(\mathbf{x}) \cdot \boldsymbol{\psi}(\mathbf{x}) d^{3} x
$$

for any two (complex) vector fields, $\zeta(\mathbf{x})$ and $\psi(\mathbf{x})$, where the integration is done over the volume, $\star$, of the unperturbed, spherically symmetric star whose density profile is $\rho_{0}(r)$. For a fluid star in the exact Newtonian formalism, $\mathcal{H}$ is $H$ ermitian with respect to this inner

3 In spherically symmetric, purely fluid stars, toroidal modes are degenerate at zero frequency. However, in rotating or magnetic stars, or in stars with a finite shear modulus, these modes have finite frequencies. 
product, i.e., $\langle\boldsymbol{\zeta} \mid \mathcal{H} \psi\rangle=\langle\mathcal{H} \zeta \mid \psi\rangle$ for any pair of sufficiently well-behaved vector fields $\boldsymbol{\zeta}, \boldsymbol{\psi}$ satisfying the boundary condition of equation (1) (Chandrasekhar 1964; Chandrasekhar \& Lebovitz 1964; Lynden-Bell \& Ostriker 1967). In fact, $\mathcal{H}$ is Hermitian for any surface boundary condition of the form

$$
c_{1} \boldsymbol{\zeta} \cdot \hat{\mathbf{r}}+\left.c_{2} \boldsymbol{\nabla} \cdot \boldsymbol{\zeta}\right|_{r=R}=0
$$

where $c_{1}$ and $c_{2}$ are constants.

Often, instead of using the full Newtonian formalism, stellar oscillation modes are calculated in the Cowling approximation (Cowling 1941), in which the Eulerian perturbations of the gravitational potential are neglected. (This reduces the problem of finding the modes from four coupled first-order differential equations to two, and it is usually a quite acceptable approximation.) For illustration and for reference in later sections, I will show here that the stellar oscillation operator is still Hermitian when this approximation is used. In this case, the mode equations take the form

$$
\mathcal{H} \boldsymbol{\xi} \equiv \nabla\left[c_{\boldsymbol{s}}^{2} \nabla \cdot \boldsymbol{\xi}+\mathbf{g} \cdot \boldsymbol{\xi}\right]+\varepsilon(\boldsymbol{\nabla} \cdot \boldsymbol{\xi}) \mathbf{g}=-\omega^{2} \boldsymbol{\xi} .
$$

(This can easily be derived, e.g., from the equations given in $\$ 2$ of Reisenegger \& Goldreich 1992.) Here, $c_{s}^{2}=(\partial p / \partial \rho)_{\text {adiabatic }}$ is the square of the adiabatic sound speed, $\mathbf{g}=-g \hat{\mathbf{r}}$ is the acceleration of gravity,

$$
\varepsilon \equiv \frac{c_{s}^{2}}{d p_{0} / d \rho_{0}}-1=\left(\frac{c_{s} N}{g}\right)^{2}
$$

is a dimensionless measure of the stable stratification, $N$ is the Brunt-Väisälä frequency (see, e.g., Reisenegger \& Goldreich 1992), and $p_{0}(r)$ and $\rho_{0}(r)$ are the equilibrium pressure and density in the star. Eq. (6) shows that, in the absence of stable stratification (i.e., if $\varepsilon=0=N^{2}$ ), the displacement fields of all modes with non-zero frequencies are gradients, and therefore irrotational. (This is still true if the Cowling approximation is not used.)

Using an integration by parts, and the spherical symmetry of the star, it is not hard to show that

$$
\begin{aligned}
\left\langle\boldsymbol{\xi}^{\prime} \mid \mathcal{H} \boldsymbol{\xi}\right\rangle-\left\langle\mathcal{H} \boldsymbol{\xi}^{\prime} \mid \boldsymbol{\xi}\right\rangle= & \int_{\star}\left[(1+\varepsilon) \rho_{0} g+c_{s}^{2} \frac{d \rho_{0}}{d r}\right]\left[\left(\nabla \cdot \boldsymbol{\xi}^{\prime}\right) \boldsymbol{\xi}-(\nabla \cdot \boldsymbol{\xi}) \boldsymbol{\xi}^{\prime}\right] \cdot \hat{\mathbf{r}} d^{3} x \\
& -\oint_{S_{\star}} \rho_{0} c_{s}^{2}\left[\left(\boldsymbol{\nabla} \cdot \boldsymbol{\xi}^{\prime}\right) \boldsymbol{\xi}-(\boldsymbol{\nabla} \cdot \boldsymbol{\xi}) \boldsymbol{\xi}^{\prime}\right] \cdot d \mathbf{s}
\end{aligned}
$$

The integral over the stellar surface, $S_{\star}$, vanishes if the vector fields satisfy a boundary condition of the form given by equation (5). The volume integral also vanishes for all $\boldsymbol{\xi}$ and $\boldsymbol{\xi}^{\prime}$ if the first term in square brackets is zero, or (using the definition of $\varepsilon$ )

$$
c_{s}^{2} \frac{d \rho_{0}}{d p_{0}}\left(\rho_{0} g-\frac{d p_{0}}{d r}\right)=0
$$


In particular, this is true if the equilibrium variables satisfy the condition of hydrostatic equilibrium,

$$
\frac{d p_{0}}{d r}=\rho_{0} g
$$

which is used in the derivation of equation (6).

This derivation can be extended, without any major modifications, to the algebraically more complicated situation where a part (or all) of the star is solid. For the modified wave equation in the presence of a finite shear modulus, see McDermott et al. (1988).

\section{SERIES EXPANSIONS AND SUM RULES}

A consequence of the Hermiticity of $\mathcal{H}$ is that the oscillation modes can be chosen orthonormal, i.e., $\left\langle\boldsymbol{\xi}_{n l m} \mid \boldsymbol{\xi}_{n^{\prime} l^{\prime} m^{\prime}}\right\rangle=\delta_{n n^{\prime}} \delta_{l l^{\prime}} \delta_{m m^{\prime}}{ }^{4}$ It is usually assumed (e.g., Cox 1980; Unno et al. 1989; Detweiler \& Ipser 1973) that the normal modes form a complete set, i.e., that any (well-behaved) vector field defined on the stellar volume can be expressed as a linear combination of the normal mode displacement fields. Although this has (to my knowledge) not been proven, it will also be assumed in the present paper.

A particularly interesting set of vector fields to expand is

$$
\mathbf{P}_{l m}(\mathbf{x})=\frac{1}{N_{l}} \nabla\left[r^{l} Y_{l m}(\theta, \phi)\right]
$$

where the constants of normalization,

$$
N_{l} \equiv\left[\frac{l(2 l+1)}{4 \pi} \int_{\star} \rho_{0}(r) r^{2(l-1)} d^{3} x\right]^{1 / 2},
$$

are chosen so that $\left\langle\mathbf{P}_{l m} \mid \mathbf{P}_{l^{\prime} m^{\prime}}\right\rangle=\delta_{l l^{\prime}} \delta_{m m^{\prime}} .{ }^{5}$ These vector fields are both divergenceless (solenoidal) and irrotational, and their linear superposition can describe, e.g., the tidal force field exerted by a binary companion (or a more complicated external mass distribution) on the star being considered. The formal expansion in terms of the modes reads

$$
\left|\mathbf{P}_{l m}\right\rangle=\sum_{n} M_{n l}\left|\boldsymbol{\xi}_{n l m}\right\rangle
$$

where the coefficients,

$$
M_{n l}=\left\langle\boldsymbol{\xi}_{n l m} \mid \mathbf{P}_{l m}\right\rangle
$$

4 That this should be the case had been pointed out, though not shown explicitly, by Cowling (1941).

5 A different normalization is used in Reisenegger \& Goldreich (1994). 
are the strengths of the multipole moments of the stellar mass distribution in the corresponding mode.

These coefficients are of physical interest, because both the energy absorbed (in the linear regime) by a mode during a tidal interaction (Press \& Teukolsky 1977; Reisenegger \& Goldreich 1994) and the damping rate of the modes due to the emission of gravitational radiation in the weak-gravity limit (Balbinski \& Schutz 1982) are proportional to $\left|M_{n l}\right|^{2}$.

Since $\mathbf{P}_{l m}(\mathbf{x})$ has the functional form of the displacement field of a spheroidal mode, it is clear that the coefficients $M_{n l}$ will vanish for all toroidal modes, implying that the latter: 1) are not excited by tidal forces, and 2) do not radiate gravitational waves (to lowest postNewtonian order). For this reason, only spheroidal modes need to be considered in the expansion.

With the normalizations chosen here, it follows from equation (13) that

$$
\sum_{n}\left|M_{n l}\right|^{2}=1
$$

for all $l$ and $m$. From this result and equation (11) of Reisenegger \& Goldreich (1994), one can derive the sum rule

$$
\sum_{n} \frac{1}{\omega_{n l}^{2 l} \tau_{n l}}=\frac{(l+1)(l+2)}{2(l-1)(2 l+1)[(2 l-1) ! !]^{2}} \frac{G}{c^{2 l+1}} \int_{\star} \rho_{0}(r) r^{2(l-1)} d^{3} x,
$$

where $\omega_{n l}$ are the frequencies of the modes and $\tau_{n l}$ are their amplitude-e-folding times due to emission of gravitational radiation in the weak-gravity limit. If the modes do not form a complete set, the ' $=$ ' sign in equations (15) and (16) should be replaced by a ' $\leq$ '.

A more general (though probably less useful) sum rule is

$$
\left\langle\mathbf{P}_{l m} \mid \mathcal{H}^{k} \mathbf{P}_{l m}\right\rangle=\sum_{n}\left(-\omega_{n l}^{2}\right)^{k}\left|M_{n l}\right|^{2}
$$

where $k$ is an arbitrary integer $\geq 0$. The right-hand side can be obtained by expanding $\mathbf{P}_{l m}$ on the left-hand side in terms of the modes, and using their orthonormality and the fact that they are eigenfunctions of $\mathcal{H}$ with eigenvalues $-\omega_{n l}^{2}$. (Eq. (15) is a special case of eq. (17), with $k=0$.)

\section{PARTICULAR MODELS}

Usually, the spheroidal modes of fluid, spherically symmetric stars can be classified according to their main restoring mechanisms into high-frequency p-modes (pressure or sound modes) and low-frequency g-modes (gravity or buoyancy modes) (Cowling 1941). For each pair of $l$ and $m$, there is an infinite set of p-modes whose frequency increases with increasing number of radial nodes, and also (for $l \geq 1$ ) an infinite set of g-modes, whose frequency decreases with increasing number of radial nodes. These two sets are separated 
by an f-mode (fundamental mode or "Kelvin mode") 6 with no radial nodes. (See Cox 1980 or Unno et al. 1989 for a more complete discussion of this classification.)

A stellar model that has been studied extensively, because its oscillation modes can be determined analytically, is the (compressible) fluid, uniform density model (see, e.g., Cox 1980; Ledoux \& Walraven 1958; and references therein). It is unphysical in the sense that all of its g-modes are unstable, but has nevertheless served as a guide for the classification and study of stellar modes. In this model, the f-mode displacement field $\boldsymbol{\xi}_{0 l m}$ is identical with the vector field $\mathbf{P}_{l m}$ defined above, ${ }^{7}$ and therefore

$$
M_{n l}=\delta_{n 0}
$$

Note that I have chosen to label the $\mathrm{f}$-mode as $n=0$, although in general it is not the lowest-frequency mode. One may, e.g., label the p-modes by positive and the g-modes by negative integers. Eq. (18) implies that the only mode that emits gravitational radiation (in the weak-field limit), and also the only mode that could be excited by a time-dependent tidal potential (in the linear regime), is the f-mode.

For fluid stellar models of nonuniform density, there are no modes with divergenceless displacement fields (Robe 1965), and therefore no mode can be identical to $\mathbf{P}_{l m}$. However, for stellar models with relatively flat density profiles, $\mathbf{P}_{l m}$ is still a very good approximation to the f-mode displacement field (cf. Chandrasekhar \& Lebovitz 1964), so that eq. (18) is still approximately true. This is made plausible by two examples, in both of which I assume, for simplicity, that the stars are neutrally stable to convection (i.e., $N^{2}=0$ ), and the Cowling approximation is used. In the first, I consider "toy" stellar models in which the density is given by $\rho_{0}(r)=\rho_{c}\left(1-\epsilon r^{2} / R^{2}\right)$, where $\rho_{c}$ is the central density, $R$ is the stellar radius, and $\epsilon$ is a dimensionless constant $(0 \leq \epsilon \ll 1)$, and the pressure, $p_{0}(r)$, is given by the condition of hydrostatic equilibrium. One can show analytically that for these models $\boldsymbol{\xi}_{0 l m} \propto \nabla\left[\left(1+\epsilon^{2} f(r)\right) r^{l} Y_{l m}(\theta, \phi)\right]$ (where $f(r)$ is a function of order unity), and $1-\left|M_{0 l}\right|^{2}$ is of order $\epsilon^{4}$, so that even models deviating appreciably from the constant density case have $\mathbf{f}$-modes nearly identical to $\mathbf{P}_{l m}$. In the second example, numerical calculations for polytropic $\left(p \propto \rho^{\gamma}\right)$ models show (Fig. 1) that $1-\left|M_{0 l}\right|^{2}$ is a rapidly decreasing function of $\gamma$, which takes values of order unity close to the stability limit $(\gamma=4 / 3)$ and approaches zero for large $\gamma$ (i.e., nearly incompressible stars). Since the fall-off is so rapid, $M_{0 l}$ is very close to unity even for models of moderate $\gamma(\sim 2)$ that could correspond, e.g., to neutron stars. The multipole moments of $\mathrm{p}$-modes and g-modes in these models are still very small, and correspondingly these modes are inefficient gravitational radiators, and they are only weakly excited by tidal fields, thus qualitatively confirming the results of Reisenegger \& Goldreich (1994).

From this, it follows that, in order to calculate meaningful numbers for $\left|M_{n l}\right|^{2}$ (and

6 This is not true for $l=1$, for which the f-mode corresponds to a uniform spatial displacement of the star, with no restoring force and zero frequency. In the Cowling approximation one does obtain a (physically meaningless) finite frequency for this mode, cf. equation (61) in Reisenegger \& Goldreich (1992).

7 This is true independently of whether the Cowling approximation is used or not. 
quantities that depend on it, such as $\tau_{n l}$ ) for modes other than the f-modes in typical neutron star models, the corresponding eigenfunctions have to be known to quite a high precision (cf. Reisenegger \& Goldreich 1992, 1994). For instance, if for such a mode one uses a displacement field $\boldsymbol{\xi}_{n l m}^{\prime}=\left(1-|\epsilon|^{2}\right)^{1 / 2} \boldsymbol{\xi}_{n l m}+\epsilon \boldsymbol{\xi}_{0 l m}$ instead of the correct displacement field $\boldsymbol{\xi}_{n l m}$, the value of $M_{n l}$ changes to $M_{n l}^{\prime}=\left(1-|\epsilon|^{2}\right)^{1 / 2} M_{n l}+\epsilon M_{0 l}$. Since $\left|M_{0 l}\right| \sim 1$, the result will be meaningless unless $|\epsilon| \ll\left|M_{n l}\right|(\ll 1)$.

\section{RELATIVISTIC EXTENSION}

Let $\mathcal{L}$ be a linear operator (Hermitian or not) whose eigenvectors span a (complex) vector space $\mathcal{S}$ in which an inner product has been defined. Then, the following two statements are equivalent:

1) Any two eigenvectors of $\mathcal{L}$ corresponding to different eigenvalues are orthogonal.

2) The functional

$$
\Lambda[\boldsymbol{\psi}]=\frac{\langle\boldsymbol{\psi} \mid \mathcal{L} \psi\rangle}{\langle\boldsymbol{\psi} \mid \boldsymbol{\psi}\rangle}
$$

is stationary with respect to arbitrary (small) variations of $\psi$ if and only if $\psi$ is an eigenvector of $\mathcal{L}$. (Of course, the value of $\Lambda[\psi]$ when $\psi$ is an eigenvector of $\mathcal{L}$ is the corresponding eigenvalue.)

In order to see that this is the case, note that, for a variation $\delta \psi$ of $\psi$, the first-order variation of $\Lambda$ is

$$
\delta \Lambda=\frac{(\langle\delta \boldsymbol{\psi} \mid \mathcal{L} \boldsymbol{\psi}\rangle+\langle\boldsymbol{\psi} \mid \mathcal{L} \delta \boldsymbol{\psi}\rangle)\langle\boldsymbol{\psi} \mid \boldsymbol{\psi}\rangle-\langle\boldsymbol{\psi} \mid \mathcal{L} \boldsymbol{\psi}\rangle(\langle\delta \boldsymbol{\psi} \mid \boldsymbol{\psi}\rangle+\langle\boldsymbol{\psi} \mid \delta \boldsymbol{\psi}\rangle)}{\langle\boldsymbol{\psi} \mid \boldsymbol{\psi}\rangle^{2}}
$$

First, assume that statement 1 is true. Write $\psi$ in terms of an orthonormal basis of $\mathcal{S}$ formed by a subset $\left\{\boldsymbol{\xi}_{i}\right\}$ of the eigenvectors of $\mathcal{L}$ as $\boldsymbol{\psi}=\sum_{i} c_{i} \boldsymbol{\xi}_{i}$. Choose $\delta \boldsymbol{\psi}=\epsilon \boldsymbol{\xi}_{k}$, where $\boldsymbol{\xi}_{k}$ is an arbitrary eigenvector, and $\epsilon$ is some small number. Then,

$$
\delta \Lambda=\frac{\lambda_{k} \sum_{i}\left|c_{i}\right|^{2}-\sum_{i} \lambda_{i}\left|c_{i}\right|^{2}}{\left(\sum_{i}\left|c_{i}\right|^{2}\right)^{2}} \operatorname{Re}\left(\epsilon^{*} c_{k}\right)
$$

where $\lambda_{i}$ is the eigenvalue corresponding to $\xi_{i}$, and Re refers to the real part of a complex number. It is clear from equation (21) that, in order for $\delta \Lambda$ to vanish for a given $k$, one must have $c_{k}=0$ unless $\lambda_{k}=\left(\sum_{i} \lambda_{i}\left|c_{i}\right|^{2}\right) /\left(\sum_{i}\left|c_{i}\right|^{2}\right) \equiv \lambda_{*}$. Since this must be true for all $k$ (which is equivalent to demanding that $\Lambda$ be stationary with respect to any small perturbation of $\psi$ ), $\psi$ is an eigenvector of $\mathcal{L}$ with eigenvalue $\lambda_{*}$. Conversely, if $\psi$ is an eigenvector of $\mathcal{L}$, then $\delta \Lambda=0$. This proves that statement 2 follows from statement 1 .

Now, assume that statement 2 is true. Choose $\boldsymbol{\psi}=\boldsymbol{\xi}_{i}$ and $\delta \boldsymbol{\psi}=\epsilon \boldsymbol{\xi}_{k}$, then $0=\delta \Lambda=$ $\epsilon\left(\lambda_{k}-\lambda_{i}\right)\left\langle\boldsymbol{\xi}_{i} \mid \boldsymbol{\xi}_{k}\right\rangle$. This implies $\lambda_{k}=\lambda_{i}$ or $\left\langle\boldsymbol{\xi}_{i} \mid \boldsymbol{\xi}_{k}\right\rangle=0$, i.e., the eigenvectors corresponding 
to different eigenvalues are orthogonal. Thus, statement 1 follows from statement 2. This proves the "theorem" stated above.

If $\mathcal{L}$ is Hermitian, then statements 1 and 2 are both true. However, neither of them is sufficient to imply the Hermiticity of $\mathcal{L}$, since both could be true, and the eigenvalues of $\mathcal{L}$ still not be real. The latter is exactly what happens in general-relativistic stellar oscillation theory. There, the modes satisfy a variational principle of the form given in equation (19) with an inner product that is a relativistic extension of that defined in equation (4) (Detweiler \& Ipser 1973). This implies that the modes are orthogonal with respect to this inner product. However, most eigenfrequencies $\omega$ are not purely real (Thorne \& Campolattaro 1967), but have a real part (corresponding to oscillations) and an imaginary part (corresponding to damping due to the emission of gravitational radiation).

\section{HYBRID MODELS FOR NEUTRON STARS}

The most extensive numerical computations of neutron star oscillation modes so far are those by the "Rochester group" and their collaborators (McDermott et al. 1988 and references therein; Strohmayer 1991; Strohmayer et al. 1991; see Finn 1987 and Reisenegger \& Goldreich 1992 for complementary work). These calculations are based on a "hybrid" approach. The stellar equilibrium model is calculated from the relativistic "OV" equations (Oppenheimer \& Volkoff 1939; see also Shapiro \& Teukolsky 1983),

$$
\begin{aligned}
& \frac{d \tilde{m}_{0}}{d \tilde{r}}=4 \pi \tilde{r}^{2} \tilde{\rho}_{0}, \\
& \frac{d \tilde{p}_{0}}{d \tilde{r}}=-\frac{G \tilde{m}_{0} \tilde{\rho}_{0}}{\tilde{r}^{2}}\left(1+\frac{\tilde{p}_{0}}{\tilde{\rho}_{0} c^{2}}\right)\left(1+\frac{4 \pi \tilde{r}^{3} \tilde{p}_{0}}{\tilde{m}_{0} c^{2}}\right)\left(1-\frac{2 G \tilde{m}_{0}}{c^{2} \tilde{r}}\right)^{-1},
\end{aligned}
$$

where $\tilde{r}$ is the radius in Schwarzschild coordinates, $\tilde{\rho}_{0}$ and $\tilde{p}_{0}$ are the local equilibrium energy density and pressure as measured in a comoving local Lorentz frame, and $\tilde{m}_{0}(\tilde{r})$ is the mass enclosed within radius $\tilde{r}$. However, the modes (the first-order perturbations of the equilibrium model) are calculated from purely Newtonian mechanics (and with the Cowling approximation). The rationale behind this choice is the following. The relativistic equilibrium equations are not much more complicated (and certainly not much harder to integrate numerically) than their (only approximately correct) Newtonian counterparts. ${ }^{8}$ However, the first-order oscillation equations and their boundary conditions are significantly more complicated in General Relativity than in the Newtonian theory, especially because the relativistic equations include both the oscillations of the star itself and the gravitational waves emitted by these oscillations. Of course, this hybrid approach is not guaranteed to be more precise than the purely Newtonian calculation, since relativistic corrections are not applied consistently, but the hope is that something is gained by using the "correct" stellar equilibrium model.

8 Furthermore, relativistic stellar models were already available to the authors from earlier evolutionary calculations. 
In using the relativistic equilibrium models together with the Newtonian oscillation equations, one must have a convention of how to translate the relativistic equilibrium quantities (energy density $\tilde{\rho}_{0}$, pressure $\tilde{p}_{0}$, enclosed mass $\tilde{m}_{0}$, and possibly other quantities, as functions of the Schwarzschild coordinate radius $\tilde{r}$ ) into the Newtonian equilibrium quantities that appear in the first-order equations. For example, the Newtonian mass density $\rho_{0}$ could be $\tilde{\rho}_{0}$ just as well as $\tilde{\rho}_{0}+\tilde{p}_{0} / c^{2}$. In the Newtonian approximation, these choices are of course all equivalent. Beyond this approximation (i.e., as far as the lowest-order post-Newtonian corrections are concerned), it may seem as if the choice were completely arbitrary, aside from the fact that some choices may give results that approximate the fully relativistic ones better then others, which seems very difficult to determine a priori. (It is not even clear in what sense the modes in the hybrid formalism are desired to approach the relativistic ones, e.g., should the frequencies approach the relativistic frequencies as measured by an inertial observer instantaneously at rest on the surface of the star, or by an observer at rest at infinity?)

In fact, however, not every choice of the first post-Newtonian correction to the equilibrium variables gives a satisfactory formalism, even if one is not concerned about the degree to which it approximates the relativistic formalism. For instance, it was shown in $\S \S 3$ and 4 that the Hermiticity of the wave operator in the purely Newtonian theory has important consequences, in particular through the orthogonality of the oscillation modes. In the fully relativistic theory, the Hermiticity is not preserved (because of the imaginary part of the eigenfrequencies that accounts for radiative damping of the modes), but the modes are still orthogonal. The "hybrid" theory does not account for the emission of gravitational waves, and therefore the frequencies should remain real. This, together with the clearly also desirable orthogonality of the modes, requires that the wave operator should be Hermitian.

In order to see how this constrains the "translation" from relativistic to Newtonian variables, consider the Newtonian oscillation operator $\mathcal{H}$ for a fluid star in the Cowling approximation, as given in equation (6). As shown in $\S 2$, this operator is Hermitian only if equation (9) is satisfied. If $\rho_{0}=$ constant, this condition is trivially fulfilled, but for more general stellar models it requires that the star be in Newtonian hydrostatic equilibrium, as given by equation (10). Thus, one natural way of performing a consistent "hybrid" computation is to take, e.g., $r=\tilde{r}, \rho_{0}(r)=\tilde{\rho}_{0}(\tilde{r})$, and $p_{0}(r)=\tilde{p}_{0}(\tilde{r})$ from the relativistic model, and define the gravitational acceleration to be used in the wave operator by equation (10). Of course, if the stellar model is calculated in the relativistic formalism, this definition of $\mathbf{g}$ implies that it will not in general satisfy the Newtonian field equation, $\nabla \cdot g=-4 \pi G \rho_{0}$, but this does not appear to affect the consistency of the formalism.

The prescription outlined above is not that followed by McDermott et al. (1988) and others, who take $r=\tilde{r}, \rho_{0}=\tilde{\rho}_{0}$, and $p_{0}=\tilde{p}_{0}$, but define $g$ in terms of the (relativistic) mass, $\tilde{m}_{0}(\tilde{r})$, enclosed within radius $\tilde{r}$, as $g=G \tilde{m}_{0}(\tilde{r}) / \tilde{r}^{2}$, as can be seen from their code, written by McDermott (1985) and kindly made available to me by Strohmayer (1993). It can be seen from the OV equations (eq. [22]) that this prescription is inconsistent with equation (10), and therefore yields a wave operator that is (slightly) non-Hermitian, and therefore has (slightly) non-orthogonal eigenfunctions. (These authors consider a partially solid star, which considerably complicates the wave operator compared to that given in 
equation (6), but does not change the argument given here.) In particular, the f-modes will not be exactly orthogonal to all other modes, which makes the gravitational-wave-damping of these other modes in their calculations quicker than it really is, as shown below.

The ratio of the gravitational acceleration calculated by the prescription suggested above to the value used by McDermott et al. (1988), calculated numerically from their models, turns out to be nearly constant $\approx 1.25$ (to within $\sim 2 \%$ ) for all $r$, except in a thin surface layer ("atmosphere") at $\tilde{\rho}_{0}<10^{10} \mathrm{~g} \mathrm{~cm}^{-3}$, whose structure was determined by a different method than that of the rest of the star. In retrospective, the nearly constant value is not entirely surprising, since this ratio (which is the combination of the three relativistic correction factors in parentheses in eqs. [22]) is $\approx\left(1-2 G \tilde{M} / c^{2} \tilde{R}\right)^{-1}$ for a star of constant $\tilde{\rho}_{0}$, with the lowest $\tilde{r}$-dependent corrections of order $\left(G \tilde{M} / c^{2} \tilde{R}\right)^{2} \sim 10^{-2}$. (Here, $\tilde{M}$ and $\tilde{R}$ are the total relativistic mass and radius of the star.)

This fact suggests that one might check the sensitivity of the modes to the precise values of $g(r)$ by multiplying $g(r)$ (as calculated by McDermott et al.) by a factor $\alpha$, recalculating the modes for several $\alpha$, and seeing how their properties evolve. This experiment, applied to the quadrupole f-mode $\left({ }_{2} f\right)$, lowest-order shear mode $\left({ }_{2} s_{1}\right)$, and crust-ocean interface mode $\left({ }_{2} i_{2}\right)$ of model NS05T8 (see McDermott et al. 1988 for details on the modes and the model), shows that the periods of the modes are not very sensitive to changes in $\alpha$ in the range $1.0 \leq \alpha \leq 1.3$, with typical changes of the order of the changes in $\alpha$. The damping time of the f-mode due to the emission of gravitational waves is also not strongly dependent on $\alpha$. However, as might have been expected, the damping times of the other two modes show a strong dependence on $\alpha$, diverging at $\alpha \approx 1.24$ (for the shear mode) and $\alpha \approx 1.26$ (for the interface mode). Of course, these divergences only mean that at those values of $\alpha$ the modes happen to be exactly orthogonal to $\mathbf{P}_{2 m}$, which is not the case for the correct modes in this model. However, the fact that these divergences exist and occur close to the parameter values corresponding to the (arguably) correct model shows that the problems pointed out above have to be taken into account in numerical computations of the damping times.

Computations with the same code used by McDermott et al. (1988), but with $g(r)$ calculated as suggested here, indicate that the true damping times are significantly longer (by factors up to $\sim 10^{3}$ and more) than those presented by these authors. However, since the numbers are probably still affected by the unphysical discontinuity introduced in their model (as indicated by still fairly non-orthogonal modes), they will not be presented here. The numerically computed values of $\left|M_{n l}\right|^{2}$ for the modes ${ }_{2} f$ and ${ }_{2} p_{1}$ in this model with the gravity prescription of $\mathrm{McDermott}$ et al. (1988) are, respectively, 0.987 and 0.042 , so that the sum rule given in equation (15) is already violated. ${ }^{9}$ This is not the case with the gravity prescription suggested here, which gives 0.985 and 0.002 .

9 The mode listed as ${ }_{2} p_{1}$ for NS05T8 in Table 5 of McDermott et al. (1988) is really a shear mode. The true ${ }_{2} p_{1}$ has a period of 0.1815 , rather than 0.1804 , milliseconds. This is the mode referred to in the text. 


\section{7: CONCLUSIONS}

A rigorous upper limit (and probably the exact value) for the sum of the squared amplitudes of the multipole moments of all modes with given $l$ and $m$ has been derived. For stellar models that are not strongly centrally condensed, the $f$-mode is the only mode contributing significantly to the sum. Thus, the multipole moments of all other modes must be comparatively small (and the sum rules give good estimates for the multipole moments of the f-modes). Therefore, modes other than the f-mode are 1) only weakly excited in any kind of tidal interactions, and 2) inefficient gravitational-wave emitters. The smallness of their multipole moments compared to that of the f-mode implies that a meaningful numerical evaluation of these properties will require a precise knowledge of the eigenfunction, in order to prevent even small $\mathrm{f}$-mode contaminations that would drastically change the results.

Such $\mathrm{f}$-mode contaminations may occur in "hybrid" computations, where Newtonian dynamical equations are used to compute the oscillations of stars whose equilibrium structure is calculated in the full general-relativistic formalism. They can be avoided by following a prescription outlined in the present paper. However, it is not clear whether anything is gained by using this hybrid approach rather than the Newtonian theory, since relativistic corrections are applied in an inconsistent and arbitrary fashion. Thus, in those cases where the full formalism of General Relativity is regarded as too complicated for practical purposes, it is probably desirable to consistently use the Newtonian theory, or some other consistent approximation to General Relativity, such as the slow-motion formalism of Finn $(1986,1987)$.

I thank Peter Goldreich for many helpful suggestions and discussions, and Tod Strohmayer for providing me with the stellar oscillation code and neutron star models of Patrick McDermott's thesis, and for helpful hints regarding their use. Correspondence with Hugh Van Horn and Kip Thorne and conversations with Patrick McDermott and Yanqin Wu are also gratefully acknowledged. This work was supported by NSF grant AST 89-13664 and NASA grant NAGW 2372 at Caltech, and by NSF grant PHY 92-45317 and a grant from the Ambrose Monell Foundation at the IAS. 


\section{REFERENCES}

Aizenman, M. L., \& Smeyers, P. 1977, Astrophys. Sp. Sci., 48, 123

Balbinski, E., \& Schutz, B. F. 1982, MNRAS, 200, 43p

Campolattaro, A., \& Thorne, K. S. 1970, ApJ, 159, 847

Chandrasekhar, S. 1964, ApJ, 139, 664

Chandrasekhar, S., \& Lebovitz, N. R. 1964, ApJ, 140, 1517

Cowling, T. G. 1941, MNRAS, 101, 367

Cox, J. P. 1980, Theory of Stellar Pulsation (Princeton Univ. Press)

Damour, T. 1987, in Gravitation in Astrophysics, B. Carter \& J. B. Hartle, eds. (New York: Plenum), 3

Detweiler, S. L., \& Ipser, J. R. 1973, ApJ, 185, 685

Fabian, A. C., Pringle, J. E., \& Rees, M. J. 1975, MNRAS, 172, 15p

Finn, L. S. 1986, MNRAS, 222, 393

Finn, L. S. 1987, MNRAS, 227, 265

Goldreich, P., \& Nicholson, P. D. 1989, ApJ, 342, 1079

Ipser, J. R., \& Thorne, K. S. 1973, ApJ, 181, 181

Jackson, J. D. 1975, Classical Electrodynamics, second edition (New York: Wiley)

Ledoux, P., \& Walraven, Th. 1958, Handbuch der Physik, ed. S. Flügge (Berlin: Springer Verlag), 51, 353

Lynden-Bell, D., \& Ostriker, J. P. 1967, MNRAS, 136, 293

McDermott, P. N. 1985, Ph. D. thesis, Univ. of Rochester (Ann Arbor: University Microfilms International)

McDermott, P. N., Van Horn, H. M., \& Hansen, C. J. 1988, ApJ, 325, 725

Oppenheimer, J. R., \& Volkoff, G. M. 1939, Phys. Rev., 55, 374

Pekeris, C. L. 1938, ApJ, 88, 189

Press, W. H., \& Teukolsky, S. A. 1977, ApJ, 213, 183

Price, R., \& Thorne, K. S. 1969, ApJ, 155, 163

Reisenegger, A., \& Goldreich, P. 1992, ApJ, 395, 240

Reisenegger, A., \& Goldreich, P. 1994, ApJ, in press

Robe, H. 1965, Bull. Acad. Roy. Belge, Cl. 5c, $5^{e}$ series, 51, 598

Shapiro, S. L., \& Teukolsky, S. A. 1983, Black Holes, White Dwarfs, and Neutron Stars (New York: Wiley)

Strohmayer, T. E. 1991, ApJ, 372, 573

Strohmayer, T., Ogata, S., Iyetomi, H., \& Ichimaru, S. 1991, ApJ, 345, 679 
Strohmayer, T. 1993, personal communication

Thomson, W. 1863, Phil. Trans. Roy. Soc. London, 153, 603

Thorne, K. S. 1969a, ApJ, 158, 1

Thorne, K. S. 1969b, ApJ, 158, 997

Thorne, K. S., \& Campolattaro, A. 1967, ApJ

Unno, W., Osaki, Y., Ando, H., Saio, H., \& Shibahashi, H. 1989, Nonradial Oscillations of Stars, second edition (Univ. of Tokyo Press)

Zahn, J. P. 1970, A\&A, 4, 452

Zahn, J. P. 1975, A\&A, 41, 329

Zahn, J. P. 1977, A\&A, 57, 383

\section{FIGURE LEGENDS}

Fig. 1: $\log \left(1-\left|M_{02}\right|^{2}\right)$ as a function of $\gamma$ for polytropic, neutrally stable stellar models with $p \propto \rho^{\gamma}$, in the Cowling approximation. $M_{02}$ is the quadrupole moment (as defined in the text) of the quadrupole $\mathrm{f}$-mode. 


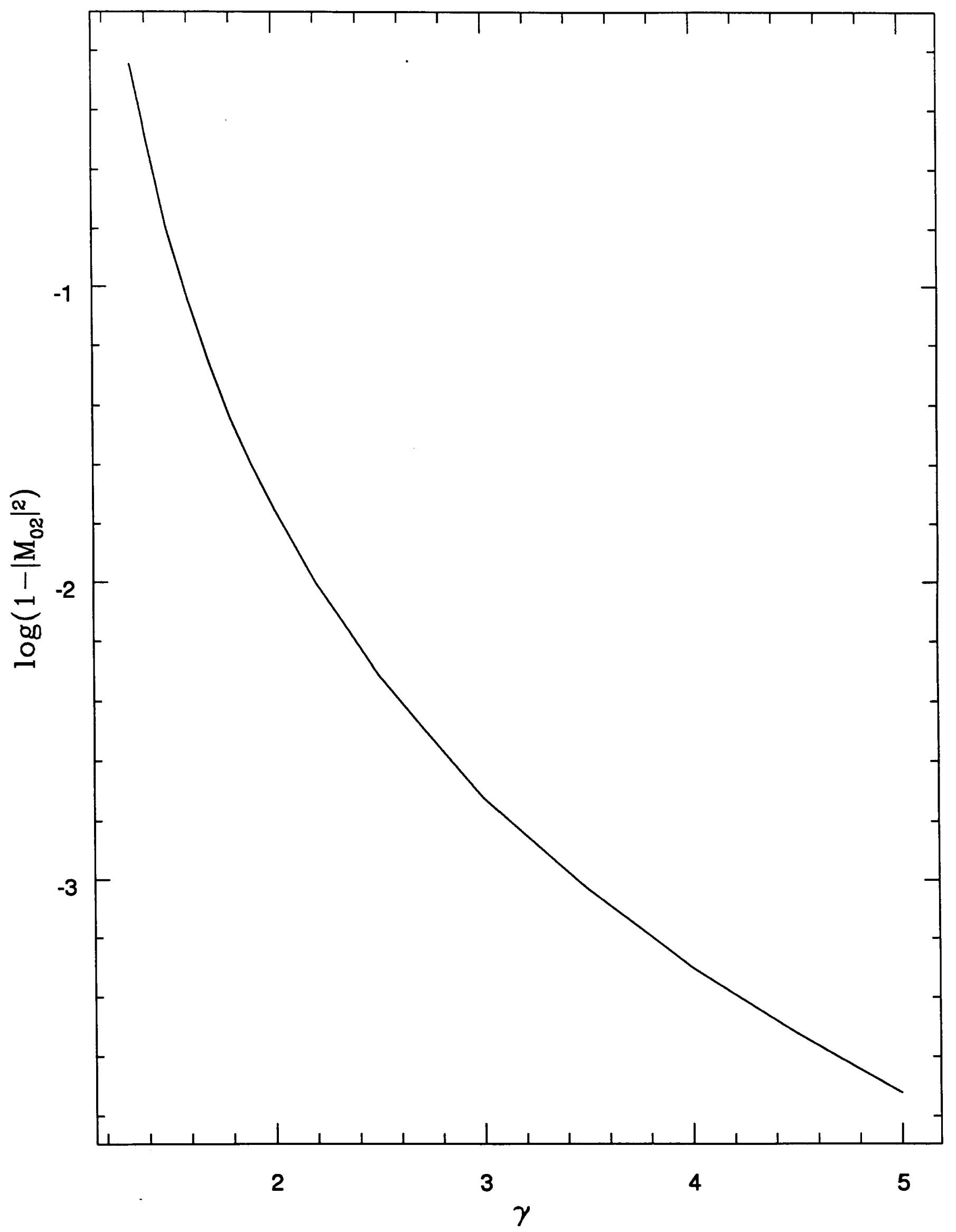


